\title{
The Effect of Acceptability and Personality on the Intention to Use Automated Vehicles among Chinese Samples
}

\author{
Weina $Q u^{1,2}$ Jing $X u^{1,2,3}$ and Yan $G e \mathbb{D}^{1,2}$ \\ ${ }^{1}$ CAS Key Laboratory of Behavioral Science, Institute of Psychology, Beijing, China \\ ${ }^{2}$ Department of Psychology, University of Chinese Academy of Sciences, Beijing, China \\ ${ }^{3}$ Tianjin College, University of Science \& Technology Beijing, Tianjin, China \\ Correspondence should be addressed to Yan Ge; gey@psych.ac.cn
}

Received 3 November 2020; Revised 8 May 2021; Accepted 15 May 2021; Published 29 June 2021

Academic Editor: Lina Kattan

Copyright (C) 2021 Weina Qu et al. This is an open access article distributed under the Creative Commons Attribution License, which permits unrestricted use, distribution, and reproduction in any medium, provided the original work is properly cited.

\begin{abstract}
The development of automated vehicles (AVs) has attracted increasing attention. Understanding public acceptance of AVs and their intention to use them, which are the primary aims of the present study, are especially important considering that increasingly more AVs will be moving on the road in the coming future. A total of 527 participants voluntarily and validly completed a series of questionnaires, including the automated vehicle acceptability scale (AVAS), Big Five Inventory (BFI), and some sociodemographic variables. The results of an internal consistency, reliability, and confirmatory factor analysis (CFA) confirmed the twofactor (contextual acceptability and impaired driving) structure of the AVAS. The Chinese public generally has a positive attitude towards AVs. In addition, our results indicate the possibility of the misuse of AVs. More importantly, the results reveal that contextual acceptability partially mediated the effect of agreeableness on the willingness to drive and the willingness to own AVs and fully mediated its effect on the willingness to rent AVs, while contextual acceptability and interest in impaired driving fully mediated the effect of the openness on the willingness to drive, own, and rent AVs. Manufacturers and retailers in the automotive vehicle industry should provide their customers with comprehensive information regarding the principles and limitations behind the system and the responsibility and obligations of the drivers to avoid misuse. Moreover, providing more targeted services according to customers' different personality traits might be a useful sales technique.
\end{abstract}

\section{Literature Review}

1.1. Brief Introduction of Automated Vehicles. With the rapid and steady development of the Chinese economy and the gradual improvement in people's living standards, motor vehicles have become an inevitable part of our daily life. Although a car provides convenience, frequent traffic accidents have become a serious social problem. Among these traffic accidents, $80 \% \sim 90 \%$ were caused by human factors [1]. Moreover, the substantial growth of vehicles causes a significant burden on urban traffic, with increasing commuting time and people's perceived decrease in life satisfaction [2]. Fortunately, automated driving will potentially contribute to overcoming these issues. For example, automatic driving technology is expected to reduce the number of traffic accidents caused by human errors, increase road fluency, allow drivers to perform other operations to improve driving comfort, increase the driving ability of the elderly and disabled [3-5], and eventually create a "safe, efficient comfortable, and energy-saving" new type of traffic mode $[6,7]$.

Based on technical capability and the role of the human driver, the American Society of Automotive Engineers (SAE) divided automated vehicles (AVs) in 2014 into 6 levels that range from level 0 to level 5, including no automation (level 0 ), driver assistance (level 1, DA), partial automation (level 2, PA), conditional automation (level 3, CA), high automation (level 4, HA), and full automation (level 5, FA), and made further specifications for this classification in $2016[8,9]$. The Chinese government proposed the "Made in China 2025" strategy to encourage innovations in the manufacturing industry and held high expectations for its development. For 
example, automated cars are expected to reduce traffic accidents by more than $80 \%$ and reduce traffic fatalities by more than $10 \%$ [10]. To achieve these goals and anticipate the market diffusion of AVs, it is especially important to understand the end-user, i.e., the public's acceptance of AVs and their consumption intention.

1.2. Public Opinions towards Automated Vehicles. Several studies have indicated that public opinions towards AVs are rather positive, but there are concerns worldwide (see, e.g., $[11,12])$. Research in Australia by the authors in [13] found that, on average, people stated a positive attitude towards AVs (the average score was above the midpoint of the scale); however, the most concerning issue was the legal issues that may occur after an AV accident and the ability to take control when wanted was the most effective way to mitigate these concerns. Research in the Netherlands by the authors in [14] revealed that public opinion is polarized on this issue; some people were very receptive to AVs, while other people did not believe that AVs provide many benefits. Software hacking and legal issues were the most common concerns. In addition, a study by the authors in [15] also indicated a diverse attitude; $39 \%$ of the comments were positive, while $23 \%$ of the comments were negative on fully AVs (the rest of the comments did not express general negativity or positivity towards automated driving and were thus tagged into other categories). Moreover, a study in France by the authors in [16] introduced a fully automated driving acceptability scale to investigate people's prior acceptability and attitudes towards fully AVs. Similarly, the results indicated that the overall attitude was positive (the average score was 4.56 on a 7-point scale); more importantly, this study emphasized the potential risk of misuse (i.e., the inadequate use of automation without recognizing its limitations).

Misuse refers to overreliance on automation and using it without recognizing its limitations, which can result in decision biases or a failure to monitor biases [17]. For example, drivers are likely to perform a task unrelated to driving when using automated driving even if they still have the responsibility to take control of the car when needed [16]. The same phenomenon was also found among $\mathrm{Ca}-$ nadian samples. Robertson et al. [18] found that male and experienced drivers reportedly were more likely to engage in other activities such as sleeping or napping and drinking and driving while using limited self-driving vehicles. However, there are no insights on this topic for the potential users of AVs in China.

In China, few studies have investigated the public acceptance of AVs. Schoettle and Sivak [19] investigated attitudes towards AVs among the public of six countries (China, Japan, India, the United Kingdom, the United States, and Australia), including 610 Chinese participants. This cross-cultural comparison found that compared with Japanese, Indian, British, American, and Australian participants, Chinese participants were most familiar with, had the most positive attitude towards, were most willing to buy, and had the fewest concerns regarding L3 and L4 AVs. However, this research mainly focused on the comparison of expectations, concerns, and willingness to pay for AVs among different countries. Xu and Fan [20] investigated the risk perceptions and insurance demand for AVs in the Chinese market, and the results found that $42 \%$ of the respondents believed that AVs could lead to reduced risk and that $45 \%$ believed that AVs could lower insurance rates. However, the underlying psychosocial factors associated with these differences, such as individual differences (i.e., age and personality), were not explored.

\subsection{The Influencing Factors of the Attitude towards Auto-} mated Vehicles. The attitude and acceptance of AVs will (potentially) influence the use of AVs; these factors influence the self-reported intention to use AVs and actual interaction with AVs. Regarding self-reported intention, the authors in [16] found that attitude and prior acceptance were useful predictors of the intention to use fully automated cars. Buckley et al. [21] showed that three dimensions of the Theory of Planned Behavior (namely, attitude towards the behavior, subjective norms, and perceived behavioral control) and one dimension of the Technology Acceptance Model (namely, ease of use) could positively predict the intended use of CA. More importantly, trust (which was measured through a questionnaire) contributed additional variance to both models. Concerning the actual interaction with [22] found that with increasing trust in automated driving, participants spent more time driving in automated drive mode. Moreover, the authors in [23] found a positive relation between trust in fully automated driving and the take-over time after an emergency take-over request in a simple practice group. This negative impact (higher levels of trust indicated a longer reaction time) might be due to overtrust in AVs.

In addition, certain socio-economic characteristics have been found to influence the willingness to use AVs [24]. Gender, age, resident status, education level, and personality traits were found to be associated with the attitude towards AVs. Researchers found that the attitudes of men, younger individuals, urban residents, and more highly educated people were more positive [13, 16, 25-27]. Additionally, people who feel more comfortable being a passenger [22] were more likely to accept an AV. Nielsen and Haustein [27] divided the Danish population into three categories, including skeptics, indifferent stressed drivers, and enthusiasts, based on their attitudes towards automated and conventional car driving and then compared the demographic variables of these three groups. People who were enthusiastic about AVs were typically male, young, highly educated, and lived in large urban areas. However, Hartwich et al. [28] found that older drivers were more positive towards AVs despite their reported lower self-efficacy in handling the system. Hohenberger [29] explored the effect of age and participants' affective reaction towards AVs on the gender differences and on the willingness to use AVs. They found that men showed more positive emotion (pleasure), less negative emotion (anxiety), and a higher level of willingness to use AVs compared with women; furthermore, 
emotion partially explained the gender difference on the usage intentions.

Considering personality, drivers who reported higher score on the driving-related sensation seeking sensationseeking were more willing to use and buy AVs [16]. Individuals with a higher score on the trait of agreeableness or conscientiousness had increased trust in automation [30]. Respondents who scored higher on neuroticism were less comfortable with the data transmission issue of AVs, while individuals who scored higher on agreeableness were less worried about this issue [14]. Few studies have investigated the public acceptance of AVs in the Chinese culture. The abovementioned study by Schoettle and Sivak was conducted in 2014; this previous study did not inspect the public's intended use of AVs and lacked an exploration of the effect of demographics and personality factors on the acceptance of AVs, which might provide more profound findings.

\subsection{The Aims and Structures of the Present Paper}

The aims of the current study are as follows:

(1) Investigating the acceptance of AVs in a sample of Chinese drivers

(2) Investigating the relation among personality, the acceptance of AVs, and the intended use of AVs

The remainder of this paper is organized as follows. Section 2 introduces the participants and discusses in detail the use of questionnaires in the present study. Section 3 provides the process used to conduct the data analysis and the main results. Section 4 discusses the statistically significant factors, the limitations, and the conclusions.

\section{Method}

2.1. Recruiting Procedure and Participants. All participants were recruited through a web-based survey company (https://www.sojump.com/). A convenience sampling technique was used while recruiting participants from social media networks and the WeChat platform because it is the most convenient and cost-effective way to recruit a relatively large number of subjects in exploratory studies. Participants were recruited only if they met the following criteria: (1) they had a valid driver's license; (2) they had actual driving experiences after they obtained their driver's license; and (3) they had heard about AVs before this survey. Qualified participants were informed that the purpose of this survey was to investigate public opinions regarding AVs. We also guaranteed that their information would be strictly confidential and only used for scientific research. Additionally, the participants were informed of their right to quit the survey at any time. After the participants agreed to take part in this survey, a series of questionnaires were presented. The questionnaires were divided into three main topics. The first part concerned the participants' acceptance of AVs, the second part involved the participants' intention towards AVs in different use cases, and the third part included the participants' personality traits and their personal information (more details of each part are introduced in the Measures section). The participants were rewarded with $20 \mathrm{RMB}$ (approximately 3.13 USD) for their participation. The study was approved by the Institutional Review Board of the Institute of Psychology, Chinese Academy of Sciences.

A total of 546 subjects completed the questionnaires. Thirteen participants were excluded due to an answering time shorter than 3 minutes (which was deemed as impossible to finish all items carefully), and additional 6 participants were excluded for selecting the same option on more than one scale. Therefore, 527 valid questionnaires from 368 males and 159 females were collected. The participants' ages ranged from 20 to 59 years $(M=36.46$ and $\mathrm{SD}=10.15)$, and their driving experiences ranged from 1 to 26 years $(M=7.86$ and $\mathrm{SD}=5.79)$. According to the statistics of the Ministry of Public Security of the People's Republic of China in 2020, $67.57 \%$ of Chinese drivers are males while $32.43 \%$ are females; $85.15 \%$ are between the age of 26 to 59 ; thus, our participants ( $70 \%$ men and $30 \%$ women; within the age of 59) roughly achieved a representative sample of Chinese drivers. More details regarding the participants' demographics are presented in Table 1.

2.2. Measures. The first part of the questionnaire is an automated vehicle acceptability scale (AVAS) to assess the prior acceptability of automated driving. The AVAS was based on the fully automated driving acceptability scale introduced by the authors in [16]. In the original study of [16], they focused on only fully automated driving, while in the present study, we did not focus on any specific level of AVs. However, we modified this scale to adjust to the Chinese language. The original scale has two factors; the first factor refers to the contextual acceptability of an AV (contextual acceptability), which contains 4 items (e.g., "the automated driving system would provide me safety compared with manual driving"), and the second factor refers to the interest in using automated driving while impaired (impaired driving), which contains 3 items (e.g., "I would delegate the driving to the automated driving system if I was over the drunk driving limit").

The Chinese version of the AVAS was translated through the following translation/back-translation procedures [31, 32]. First, three psychology students translated all items into Chinese independently. Second, two psychology professors who have long engaged in the study of traffic safety compared the three translations and formed a draft version that was more fluent and accurate. Third, a professional English-Chinese translator back-translated the draft version into English and compared it with the original items to affirm that the translation did not alter the meaning of the original version. Fourth, five students were invited to ensure that all items were understandable, and the final version was then formed.

The Chinese version of the AVAS asked the participants to indicate the degree to which they agreed with certain descriptions about AVs on a 7-point Likert scale from 1 = "I strongly disagree" to $7=$ "I strongly agree." Items 1 and 4 were reversed coded. The total scores for each subscale were 
TABle 1: Participant demographics $(N=527)$.

\begin{tabular}{|c|c|c|}
\hline & $n$ & Percent (\%) \\
\hline \multicolumn{3}{|c|}{ Age groups by gender } \\
\hline \multicolumn{3}{|c|}{ 20-30 years old } \\
\hline Male & 131 & 24.9 \\
\hline Female & 79 & 15.0 \\
\hline \multicolumn{3}{|l|}{$31-40$ years old } \\
\hline Male & 81 & 15.4 \\
\hline Females & 29 & 5.5 \\
\hline \multicolumn{3}{|l|}{$41-50$ years old } \\
\hline Male & 101 & 19.1 \\
\hline Female & 28 & 5.3 \\
\hline \multicolumn{3}{|l|}{$51-59$ years old } \\
\hline Male & 55 & 10.4 \\
\hline Female & 23 & 4.4 \\
\hline \multicolumn{3}{|c|}{ Number of years after holding a driver's license } \\
\hline$<3$ years & 81 & 15.4 \\
\hline $3-5$ years & 164 & 31.1 \\
\hline $6-10$ years & 158 & 30.0 \\
\hline $11-20$ years & 106 & 20.1 \\
\hline$>20$ years & 18 & 3.4 \\
\hline \multicolumn{3}{|c|}{ Annual mileage $(\mathrm{km})$} \\
\hline$\leq 10,00$ & 83 & 15.7 \\
\hline $10,01-10,000$ & 245 & 46.5 \\
\hline $10,001-50,000$ & 185 & 35.1 \\
\hline$>50,000$ & 14 & 2.7 \\
\hline \multicolumn{3}{|c|}{ Weekly mileage $(\mathrm{km})$} \\
\hline$\leq 50$ & 75 & 14.2 \\
\hline $51-150$ & 140 & 26.6 \\
\hline $151-400$ & 212 & 40.2 \\
\hline$>400$ & 100 & 19.0 \\
\hline
\end{tabular}

calculated; higher scores indicated more acceptance of AVs and more interest in using AVs while impaired.

The second part of the questionnaire was designed to measure the intention to use automated driving, which was measured through the following items: "I am willing to drive an automated vehicle"; "I am willing to own an automated vehicle"; and "I am willing to rent an automated vehicle." The participants were asked the degree to which they agreed with certain descriptions on a 6-point Likert scale that ranged from $1=$ "I strongly disagree" to $6=$ "I strongly agree." Higher scores indicate a greater intention to use AVs $[13,19]$.

The third part of the questionnaire included the participants' personality traits and their personal information. Personality was measured through the Chinese version of the Big Five Inventory (BFI), which was created by the authors in [33]; it comprises 44 items and is used to measure the Big Five personality model [34]. The scale includes the following factors: extroversion (8 items, being energetic or sociable); agreeableness (9 items, being friendly or helpful); conscientiousness ( 9 items, being reliable); neuroticism (8 items, being anxious or irritable); and openness (10 items, being curious and seeking new experiences). The participants were asked to indicate the degree to which the items describe them by using a Likert scale that ranged from strongly disagree (1) to strongly agree (5), and the total scores for each subscale were calculated.
The participants were also asked to provide common information about their sociodemographic variables, such as age, gender, level of education, and number of years after obtaining a driver's license. In addition, the participants were asked about how familiar they are with AVs on a scale that ranged from 1-completely unfamiliar to 7-completely familiar.

\section{Results}

3.1. Descriptive Statistics. Table 2 presents the means and standard deviations and distribution of the responses to each item in the AVAS. The item-total correlations (ITCs) of each item are also reported. The mean values showed that 6 out of 7 items were above 4 (the median point of the 7 point Likert scale), indicating that most participants are willing to use the automated driving system after they were bored, had passengers, drunk, tired, or took medicine; and they considered automated driving system safe. The ITCs ranged from 0.67 to 0.93 and reached high levels. Specifically, $93.3 \%$ of the Chinese individuals scored above 4 on the interest in using AVs while impaired dimension, 36.1\% of the respondents strongly agreed that they would choose to use an automated driving system if they were over the drunk driving limit, and $36.2 \%$ of the respondents strongly agreed that they would choose to use an automated driving system if they took medication that affected their ability to drive. In addition, $57.5 \%$ of the respondents chose 4 points and below when asked about how familiar they are with AVs.

3.2. AVAS Reliability. The means, standard errors, ranges, and Cronbach's $\alpha$ of the AVAS and BFI are listed in Table 3. The internal consistency reliability should be considered to be adequate and fall within an acceptable range for the following Cronbach's $\alpha$ coefficients: 0.65 (Contextual acceptability); 0.92 (Impaired driving); 0.81 (Extroversion); 0.74 (Agreeableness); 0.83 (Conscientiousness); 0.81 (Neuroticism); and 0.81 (Openness). Table 3 also shows the mean values of the three items (i.e., "willingness to drive," "willingness to use," and "willingness to rent"); all items were higher than 4 (the median point of 7-point Likert scale).

3.3. Confirmatory Factor Analysis. A confirmatory factor analysis (CFA) was conducted to evaluate the two-factor structure of the AVAS. The statistical program AMOS 21.0 was used. The chi-square statistic $\left(x^{2}=84.21, \mathrm{df}=12\right.$, and $p=0.00$ ) was significant. Five commonly used goodness-offit indices were employed to assess the model fit. The Joreskog-Sorbom goodness-of-fit index $=0.96 \quad(\mathrm{GFI} \geq 0.90)$, Bentler's comparative fit index $=0.96(\mathrm{CFI} \geq 0.90)$, the incremental fit index $=0.96$ (IFI $\geq 0.90$ ), the Tucker-Lewis index $=0.93(\mathrm{TLI} \geq 0.90)$, and the root mean square error of approximation $=0.11($ RMSEA $\leq 0.08)$ which indicated that the tested model fits the data well $[35,36]$. The results are presented in Figure 1. 
TABLE 2: Response means, standard deviations, and distributions.

\begin{tabular}{|c|c|c|c|c|c|c|c|c|c|}
\hline \multirow{2}{*}{ AVAS items } & \multirow{2}{*}{$\mathrm{M}(\mathrm{SD})$} & \multirow{2}{*}{ ITCs } & \multicolumn{7}{|c|}{ Response distributions (\%) } \\
\hline & & & 1 & 2 & 3 & 4 & 5 & 6 & 7 \\
\hline \multicolumn{10}{|l|}{ Contextual acceptability } \\
\hline $\begin{array}{r}\text { I would rather keep manual control of my vehicle } \\
\text { the automated driving system on ev }\end{array}$ & $\begin{array}{c}4.81 \\
(1.13)\end{array}$ & $0.73^{* *}$ & 0.6 & 4.4 & 5.5 & 21.1 & 46.1 & 16.5 & 5.9 \\
\hline $\begin{array}{l}2 \text { The automated driving system would provide me safety compared with } \\
\text { manual driving }\end{array}$ & $\begin{array}{c}4.39 \\
(1.17)\end{array}$ & $0.73^{* *}$ & 0.8 & 2.7 & 18.8 & 33.2 & 28.1 & 12.3 & 4.2 \\
\hline $\begin{array}{r}\text { If driving was boring to me, I would rather } \\
\text { driving system instead of doin }\end{array}$ & 5. & $0.67^{* *}$ & 0.8 & 0.8 & 5.5 & 6.3 & 46.5 & 27.3 & 12.9 \\
\hline $\begin{array}{l}\text { If I had passengers in my automated car, I would rather drive by myself than } \\
\text { delegating it to the automated driving system }\end{array}$ & $\begin{array}{c}3.71 \\
(1.30)\end{array}$ & $0.74^{* *}$ & 4.0 & 12.5 & 30.7 & 23.0 & 23.0 & 4.7 & 2.1 \\
\hline \multicolumn{10}{|l|}{ Impaired driving } \\
\hline 5 I would delegate the driving to th & $\begin{array}{l}5.83 \\
(1.18)\end{array}$ & $0.93^{* *}$ & 0.8 & 1.1 & 2.7 & 4.2 & 28.7 & 26.6 & 36.1 \\
\hline 6 I would delegate the driving to the automated driving system if I was ti & $\begin{array}{c}5.87 \\
(1.06)\end{array}$ & $0.90^{* *}$ & 0.6 & 1.1 & 1.3 & 2.3 & 28.3 & 35.1 & 31.3 \\
\hline $\begin{array}{l}\text { I would delegate the driving to the automated driving system if I took } \\
\text { medication that affected my ability to drive }\end{array}$ & $\begin{array}{c}5.90 \\
(1.15)\end{array}$ & $0.93^{* *}$ & 1.1 & 1.1 & 1.7 & 2.3 & 26.0 & 31.5 & 36.2 \\
\hline
\end{tabular}

TABle 3: Descriptive statistics for all the scales.

\begin{tabular}{lccccc}
\hline & Item & Mean & SD & Range & Cronbach's $\alpha$ \\
\hline AVAS & 7 & & & & \\
Contextual & 4 & 4.55 & 0.84 & $1-7$ & 0.69 \\
acceptability & 3 & 5.87 & 1.04 & $1-7$ & 0.91 \\
Impaired driving & 44 & & & & \\
BFI & 8 & 3.37 & 0.66 & $1-5$ & 0.81 \\
Extroversion & 9 & 3.91 & 0.49 & $1-5$ & 0.74 \\
Agreeableness & 9 & 3.79 & 0.58 & $1-5$ & 0.83 \\
Conscientiousness & 8 & 2.54 & 0.66 & $1-5$ & 0.81 \\
Neuroticism & 10 & 3.63 & 0.53 & $1-5$ & 0.81 \\
Openness & 1 & 4.67 & 0.93 & $1-7$ & \\
Willingness to drive & 1 & 4.72 & 0.99 & $1-7$ & \\
Willingness to own & 1 & 4.40 & 1.00 & $1-7$ & \\
Willingness to rent & & & & & \\
\hline
\end{tabular}

3.4. Correlations among the AVAS, BFI Factors, and Other Measured Variables. The Spearman correlations among the variables are presented in Table 4 . The correlation coefficient between contextual acceptability and impaired driving was 0.44 . For the relation between the AVAS and the sociodemographic variables, the results indicated a significant correlation between contextual acceptability and only age. For the relation between the AVAS and BFI, the results indicated that contextual acceptability was positively correlated with extroversion, agreeableness, conscientiousness, and openness and negatively correlated with neuroticism. Moreover, impaired driving showed a similar trend and was positively correlated with agreeableness, conscientiousness, and openness. For the relation between the AVAS and the intention to use AVs, the results indicated that both contextual acceptability and impaired driving were positively correlated with the willingness to drive, own, and rent an AV.

3.5. Path Analysis. Maximum likelihood procedures were used to test the fit of the path model. The statistical program AMOS 21.0 was used. After deleting all nonsignificant pathways among the Big Five personality traits, AVAS, and intentions, the revised model showed a good model fit. The chi-square statistic $\left(x^{2}=12.40, \mathrm{df}=5\right.$, and $\left.p=0.03\right)$ was significant. Five commonly used goodness-of-fit indices were employed to assess the model fit. The Joreskog-Sorbom goodness-of-fit index $=0.99$ (GFI $\geq 0.90$ ), Bentler's comparative fit index $=0.99(\mathrm{CFI} \geq 0.90)$, the incremental fit index $=0.99 \quad(\mathrm{IFI} \geq 0.90)$, the Tucker-Lewis index $=0.97$ $(\mathrm{TLI} \geq 0.90)$, and the root mean square error of approximation $=0.05$ (RMSEA $\leq 0.08)$ which indicated that the tested model fits the data well $[36,37]$. The results are presented in Figure 2.

First, the results indicated that contextual acceptability mediated the effect of agreeableness and openness on the willingness to drive, willingness to own, and willingness to rent AVs. Second, the results suggested that impaired driving mediated the effect of openness on the willingness to drive, willingness to own, and willingness to rent. Third, the results indicated that agreeableness had a direct effect on the willingness to drive and willingness to own. After deleting all nonsignificant pathways among the Big Five personality traits, the AVAS, and intentions, using the maximum likelihood procedures in the AMOS 21.0 program and selecting indirect/direct and total effects as outputs, the values of the standardized effects are shown in Table 5.

\section{Discussion}

The primary aims of the present study were to investigate the user's acceptance of AVs and to explore the influence of personality and acceptance on the intention to use AVs. The results indicate that contextual acceptability and impaired driving as measured by the Chinese version of the AVAS are important determinants of the acceptance of AVs for potential Chinese users. Our results also reveal an overall positive attitude towards AVs and interest in impaired driving among the Chinese public. More importantly, interest in impaired driving partially mediated the effect of 


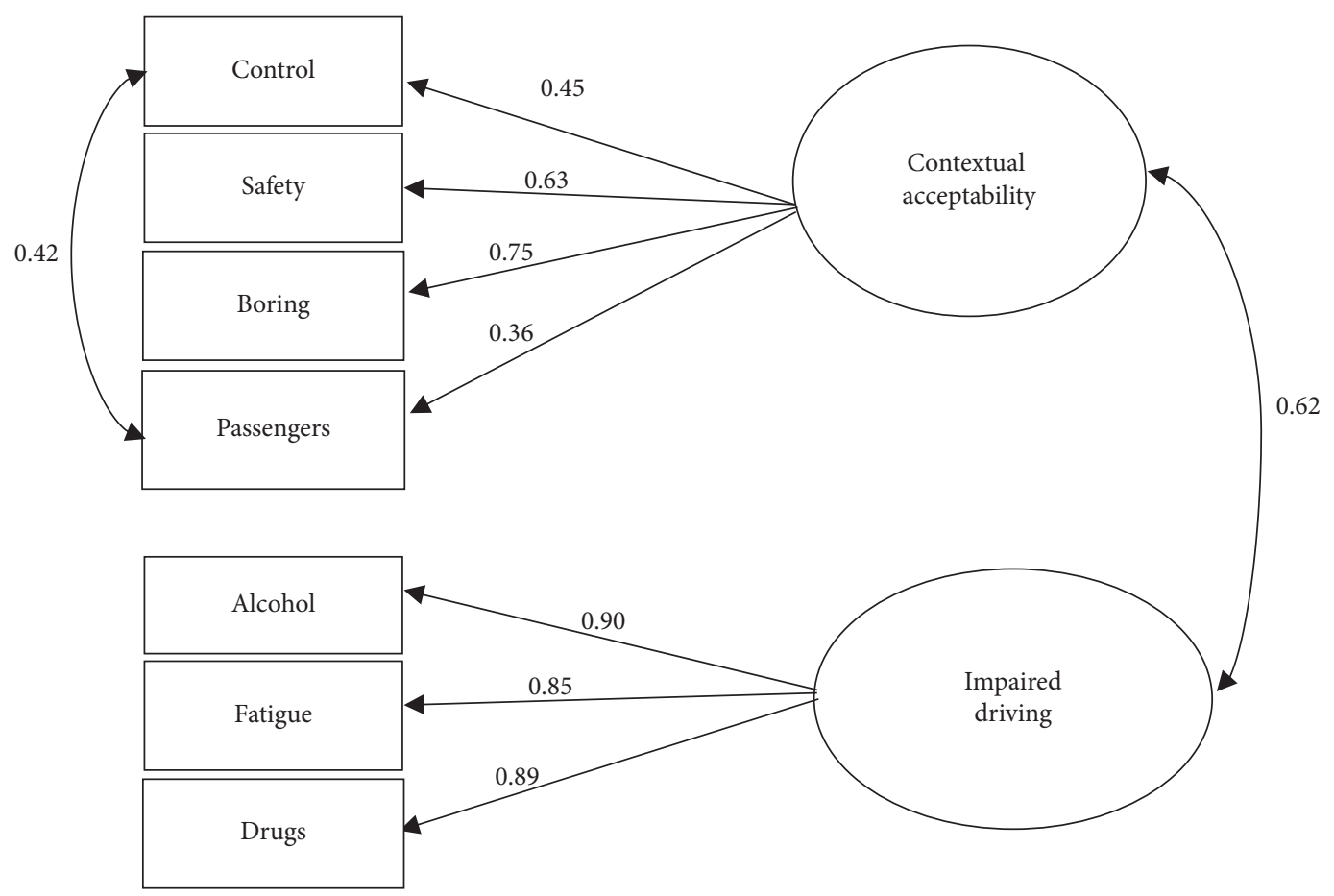

FIGURE 1: Results of the structural equation model on the AVAS with significantly standardized regression estimates.

agreeableness on the willingness to drive and willingness to own AVs and fully mediated its effect on the willingness to rent AVs, while contextual acceptability fully mediated the effect of openness on the willingness to drive, own, and rent AVs.

The internal consistency reliability of the Chinese version of the AVAS was confirmed by the high Cronbach's $\alpha$ coefficients, and the structure validity was confirmed by the confirmatory factor analysis. In addition, the two factors (contextual acceptability and impaired driving) of the scale were suitable for Chinese drivers. Contextual acceptability involves favorable conditions that would lead drivers to accept automated driving; for example, if drivers felt bored or if they were accompanied by passengers while driving, more than half of the participants would use an automated driving system instead of manual driving in these cases. This finding is reasonable because most of the public expect AVs to drive more safely than when manually driven, and they expect AVs to help relieve drivers from driving tasks, which opens the possibility of performing other tasks while traveling $[13,14,19,27]$.

More importantly, we should pay close attention to the majority of the respondents who were interested in using an automated driving system while impaired, such as when they are drunk or tired, on which occasions they are forbidden or not recommended to drive manually. Consistent with a previous French study [16] and a Canadian study [18], this issue could lead to the potential risk of the misuse of AVs. For example, $36.1 \%$ of the respondents strongly agreed that they would choose to use an automated driving system if they were over the drunk driving limit; however, this choice would not be completely safe until at least L4 automation is available because at lower levels of automation, drivers still have the obligation or responsibility to take control of the car when needed $[8,9]$. Thus, it is necessary for drivers to remain conscious and retain the ability to drive even if they are using the automated driving system, which drunk drivers are not qualified to do; it would be dangerous and illegal for drunk drivers to drive even a small distance as their driving performance is impaired by alcohol [38-41]. This potential misuse might partially be due to the lack of knowledge about AVs among the public; they are unaware of their responsibility and the limitations of the automation system. We found that $57.5 \%$ of the respondents chose 4 points and below when asked about how familiar they are with AVs. This finding indicates that the public is not very familiar with AVs. Furthermore, $93.3 \%$ of Chinese individuals scored above 4 on the interest in using AVs while impaired dimension, which was higher than the $70.6 \%$ of French individuals [16]. On the one hand, due to the heavily penalization in China for drink and driving, Chinese individuals might be more cautious when it comes to drink and driving, thus intending to turn to automated driving system for help. On the other hand, this finding might indicate a more severe problem for misuse among Chinese individuals and might result from the more extreme traffic load conditions in China than in other countries. With the annually increasing number of motor vehicles and the expanding road network, China currently confronts more severe road trauma $[42,43]$, which leads to a major burden for urban commuters [2]. Longer regular commuting times increase the acceptance of and the willingness to use AVs [25].

The results of the correlation show that contextual acceptance and impaired driving both positively correlated with the intended use of AVs. It is reasonable that the 


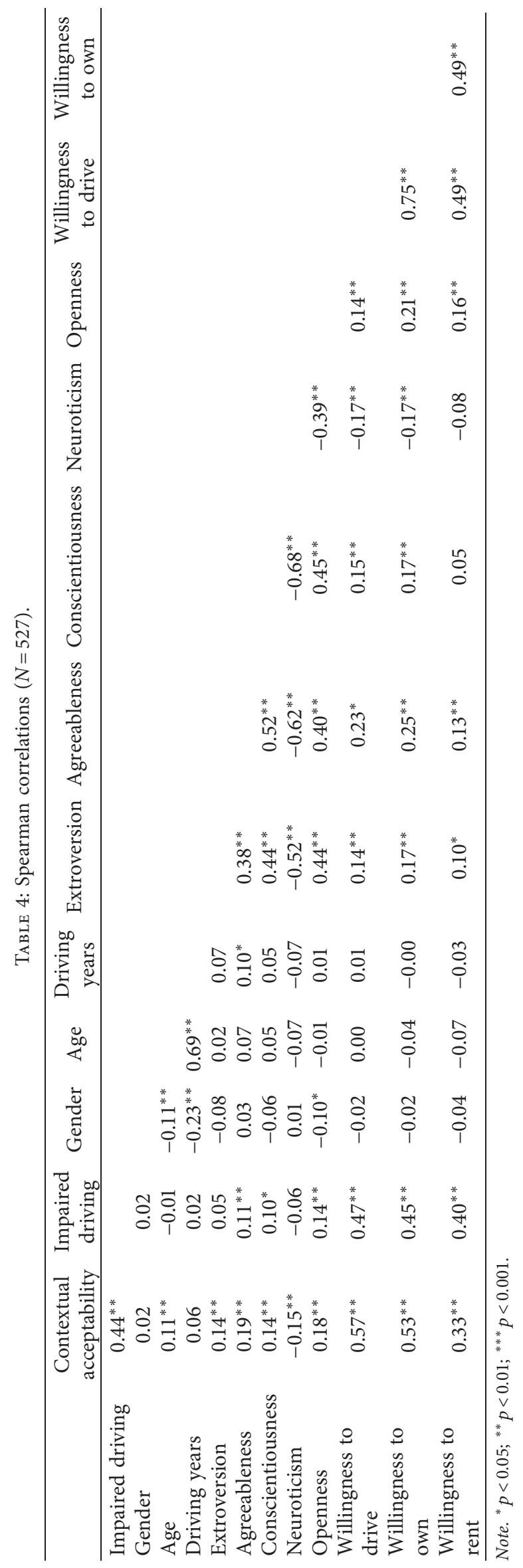




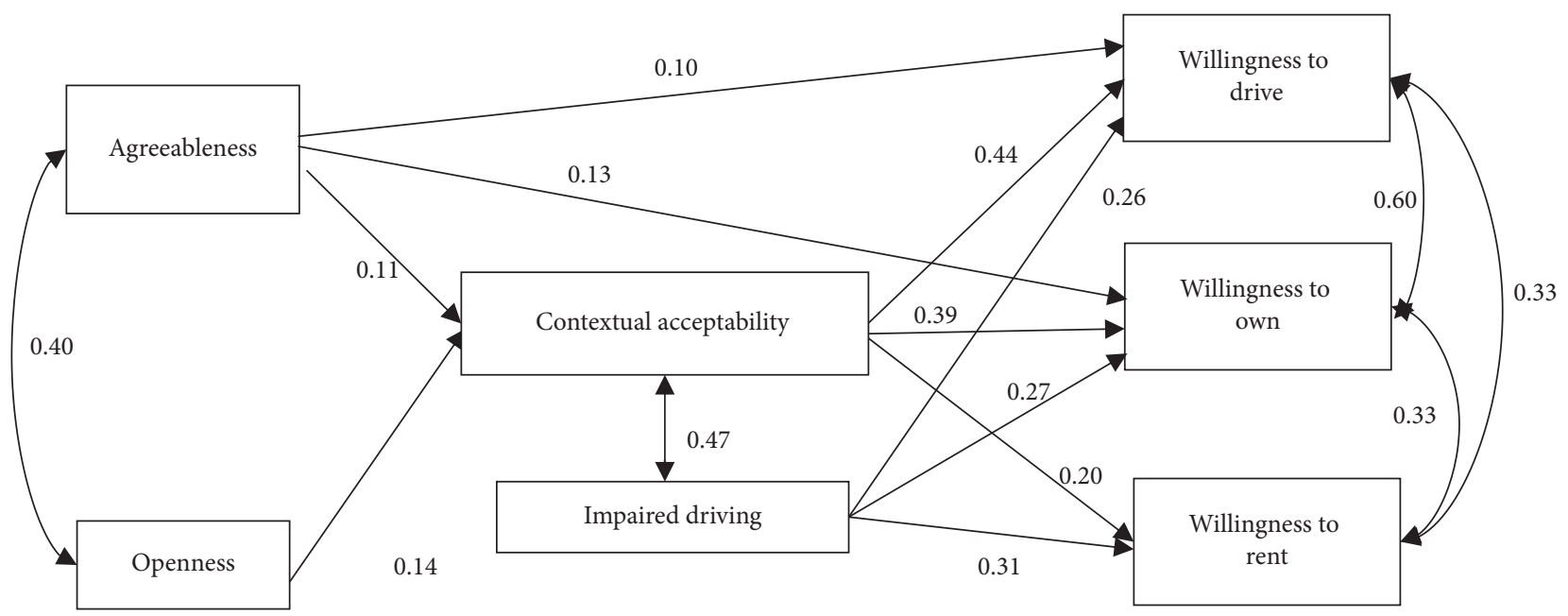

FIGURE 2: Revised path model of the variables with significantly standardized regression estimates.

TABLE 5: Standardized effects from the path analysis.

\begin{tabular}{lccc}
\hline & Total effect & Direct effect & Indirect effect \\
\hline Agreeableness $\longrightarrow$ willingness to drive & 0.15 & 0.10 & 0.05 \\
Agreeableness $\longrightarrow$ willingness to own & 0.17 & 0.13 & - \\
Agreeableness $\longrightarrow$ willingness to rent & 0.02 & - & 0.04 \\
Openness $\longrightarrow$ willingness to drive & 0.10 & - & 0.02 \\
Openness $\longrightarrow$ willingness to own & 0.09 & - & 0.10 \\
Openness $\longrightarrow$ willingness to rent & 0.07 & & 0.09 \\
\hline
\end{tabular}

participants who agreed more with the use of AVs in multiple situations and the use of AVs while impaired are more likely to drive, buy, and rent AVs. This finding is consistent with a previous study [16]. No relationships were found between gender and the acceptance or intended use of AVs. In addition, age had a slight positive relation to contextual acceptability although many researchers have found that males and younger individuals tend to have more positive attitudes towards AVs than females and elderly individuals tend to have $[13,26,27]$.

The effects of personalities on the intention to use AVs were mediated by acceptability. The results reveal that the effect of agreeableness on the willingness to drive and willingness to own AVs was partially mediated by contextual acceptability, while the effect of agreeableness on the willingness to rent was fully mediated by contextual acceptability. The direct effect of agreeableness on the willingness to drive and own AVs might be because the participants who generally scored higher on agreeableness tend to be more altruistic and friendly [44] and do not believe that AVs are silly [14]. Thus, these individuals are more willing to drive and own an AV by themselves. The indirect effect of agreeableness on the intended use of AVs revealed that the participants who scored higher on agreeableness are more willing to use AVs in different cases. The effect of openness on the willingness to drive, own, and rent AVs was fully mediated by contextual acceptability and interest in impaired driving, which means that the participants who scored higher on openness were more generally accepting of AVs. This result may be because these individuals are more curious (interested in new things) and adventurous (openminded and seeking new experiences) [45]. The automotive industry is currently undergoing a potentially revolutionary change that has attracted much attention [13], but AVs remain a novel development for most of the public. Individuals with an openness personality trait are most likely to be open-minded and try new things, such as an automated driving system; thus, they have the intention to use AVs in different use cases.

Several limitations remain in the present study. First, we asked the participants to evaluate the degree to which they agreed with some descriptions about automated vehicles without providing any definitions or introductions of $\mathrm{AV}$. This might result in the subjects evaluating their imagination of AV rather than evaluating the actual existing commodity. The advantage of this design is that it makes it possible to reveal the true expectations of the public for AVs; however, the disadvantage is that the imagination may vary among persons and thus lead to inconsistent evaluation standards. Next, all data were collected through self-reported questionnaires on the Internet, which may limit our participants to people who have access to the Internet. An on-site questionnaire is a good way to ameliorate this defect. Lastly, the majority of subjects were younger and middle-aged (only $4.6 \%$ of subjects were above 40 years old). Thus, generalizing the results should be performed with caution, and future studies should focus more on older subjects.

Accordingly, the results of the internal consistency, reliability, and CFA confirmed the two-factor (contextual acceptability and impaired driving) structure of the AVAS, 
which is consistent with the structure proposed by [16]. The present study reveals that, firstly, the Chinese public generally has a positive attitude towards AVs. Secondly, our study indicates the possibility of misuse of AVs among Chinese drivers. Manufacturers and retailers in the automotive industry should provide their customers with comprehensive information regarding the principles and limitations behind the system and the responsibility and obligations of the drivers to prevent drivers from misusing the system, which may lead to an accident. In addition, considering the points of view of policy and practice, it might be helpful for countries to enact laws to regulate the use of different levels of AVs; for example, it is also necessary to prohibit to at least an L4 automation drinking while driving or using mobile phones while driving because at lower levels of automation because drivers still have the obligation or responsibility to drive AVs like normal cars. Furthermore, contextual acceptability partially mediated the effect of agreeableness on the willingness to drive and willingness to own AVs and fully mediated its effect on the willingness to rent AVs, while contextual acceptability and interest in impaired driving fully mediated the effect of openness on the willingness to drive, own, and rent AVs. It would be helpful for automotive vehicle manufacturers and retailers to provide more targeted services according to the different personality traits of customers.

\section{Data Availability}

The data used to support the findings of this study are available from the corresponding author upon request.

\section{Conflicts of Interest}

The authors declare that they have no conflicts of interest.

\section{Authors' Contributions}

Weina Qu was conceptualized the study, developed methodology, and wrote, reviewed, and edited the manuscript. Jing $\mathrm{Xu}$ was responsible for data collection and prepared original draft. Yan Ge conceptualized the study and reviewed and edited the manuscript.

\section{Acknowledgments}

This study was partially supported by the National Natural Science Foundation of China (grant nos. 32071064, 32071066, 31771225, U1736220, and 71971073).

\section{Supplementary Materials}

This table presents the corresponding English and Chinese version of the automated vehicle acceptability scale that we used and translated in the present study. This is the English version of the whole surveys that we used in the present study, including three parts of information that we asked the participants to provide. (Supplementary Materials)

\section{References}

[1] D. Parker, J. T. Reason, M. Asr, and S. G. Stradling, "Driving errors, driving violations and accident involvement," Ergonomics, vol. 38, no. 5, pp. 1036-1048, 1995.

[2] P. Nie and A. Sousa-Poza, Commute Time and Subjective Well-Being in Urban China, China Economic Review, London, UK, 2016.

[3] J. M. Anderson, N. Kalra, K. D. Stanley, P. Sorensen, C. Samaras, and O. A. Oluwatola, Autonomous Vehicle Technology: A Guide for Policymakers, RAND Corporation, Santa Monica, CA, USA, 2014.

[4] D. J. Fagnant and K. Kockelman, "Preparing a nation for autonomous vehicles: opportunities, barriers and policy recommendations," Transportation Research Part A, vol. 77, pp. 167-181, 2015.

[5] C. Mui and P. B. Carroll, Driverless Cars. Trillions are up for Grabs, Cornerloft Press, Seattle, WA, USA, 2013.

[6] M. Kyriakidis, J. C. F. D. Winter, N. Stanton et al., "A human factors perspective on automated driving," Theoretical Issues in Ergonomics Science, vol. 20, no. 3, 2018.

[7] W. Yunqiang, "Thinking about self-driving vehicles related problem," China Public Security. Academy Edition, vol. 9, no. 6, pp. 16-17, 2017.

[8] SAE International, Taxonomy and Definitions for Terms Related to On-Road Motor Vehicle Automated Driving Systems, SAE International, Washington, DC, USA, 2014.

[9] SAE International, Taxonomy and Definitions for Terms Related to Driving Automation Systems for On-Road Motor Vehicles, SAE International, Washington, DC, USA, 2016.

[10] National Manufacturing Strategic Advisory Committee, Made in China 2025, Publishing House of Electronics Industry, Beijing, China, 2016.

[11] E. Fraedrich, D. Heinrichs, F. J. Bahamonde-Birke, and R. Cyganski, "Autonomous driving, the built environment and policy implications," Transportation Research Part A Policy \& Practice, vol. 122, pp. 162-172, 2018.

[12] D. M. Sanbonmatsu, D. L. Strayer, Z. Yu, F. Biondi, and J. M. Cooper, "Cognitive underpinnings of beliefs and confidence in beliefs about fully automated vehicles," Transportation Research Part F Traffic Psychology \& Behaviour, vol. 55, pp. 114-122, 2018.

[13] M. König and L. Neumayr, "Users' resistance towards radical innovations: the case of the self-driving car," Transportation Research Part F: Traffic Psychology and Behaviour, vol. 44, pp. 42-52, 2017.

[14] M. Kyriakidis, R. Happee, and J. C. F. D. Winter, "Public opinion on automated driving: results of an international questionnaire among 5000 respondents," Transportation Research Part F Traffic Psychology \& Behaviour, vol. 32, pp. 127-140, 2015.

[15] P. Bazilinskyy, M. Kyriakidis, and J. D. Winter, "An international crowdsourcing study into people's statements on fully automated driving is," Procedia Manufacturing, vol. 3, pp. 2534-2542, 2015.

[16] W. Payre, J. Cestac, and P. Delhomme, "Intention to use a fully automated car: attitudes and a priori acceptability," Transportation Research Part F Psychology \& Behaviour, vol. 27, pp. 252-263, 2014.

[17] R. Parasuraman and V. Riley, "Humans and automation: use, misuse, disuse, abuse," Human Factors, vol. 39, no. 2, pp. 230-253, 1997.

[18] R. D. Robertson, S. R. Meister, W. G. M. Vanlaar, and M. M. Hing, "Automated vehicles and behavioural adaptation 
in Canada," Transportation Research Part A Policy \& Practice, vol. 104, pp. 50-57, 2017.

[19] B. Schoettle and M. Sivak, Public Opinion about Self-Driving Vehicles in China, India, Japan, the U.S., the U.K., and Australia, University of Michigan Ann Arbor Transportation Research Institute, Ann Arbor, MI, USA, 2014.

[20] X. Xu and C. K. Fan, "Autonomous vehicles, risk perceptions and insurance demand: an individual survey in China," Transportation Research Part A Policy \& Practice, vol. 124, pp. 549-556, 2018.

[21] L. Buckley, S. A. Kaye, and A. K. Pradhan, "Psychosocial factors associated with intended use of automated vehicles: a simulated driving study," Accident; Analysis and Prevention, vol. 115, p. 202, 2018.

[22] L. J. Molnar, L. H. Ryan, A. K. Pradhan, D. W. Eby, R. M. S. Louis, and J. S. Zakrajsek, "Understanding trust and acceptance of automated vehicles: an exploratory simulator study of transfer of control between automated and manual driving," Transportation Research Part F Traffic Psychology \& Behaviour, vol. 58, pp. 319-328, 2018.

[23] W. Payre, J. Cestac, and P. Delhomme, "Fully automated driving: impact of trust and practice on manual control recovery," Human Factors, vol. 58, no. 2, 2016.

[24] F. Becker and K. W. Axhausen, Literature Review on Surveys Investigating the Acceptance of Automated Vehicles, Transportation, London, UK, 2017.

[25] C. J. Haboucha, R. Ishaq, and Y. Shiftan, "User preferences regarding autonomous vehicles," Transportation Research Part C Emerging Technologies, vol. 78, pp. 37-49, 2017.

[26] L. M. Hulse, H. Xie, and E. R. Galea, "Perceptions of autonomous vehicles: relationships with road users, risk, gender and age," Safety Science, vol. 102, pp. 1-13, 2018.

[27] T. A. S. Nielsen and S. Haustein, "On sceptics and enthusiasts: what are the expectations towards self-driving cars?" Transport Policy, vol. 66, pp. 49-55, 2018.

[28] F. Hartwich, C. Witzlack, M. Beggiato, and J. F. Krems, "The first impression counts - a combined driving simulator and test track study on the development of trust and acceptance of highly automated driving," Transportation Research Part F Traffic Psychology \& Behaviour, vol. 65, pp. 522-535, 2018.

[29] C. Hohenberger, M. Spörrle, and I. M. Welpe, "How and why do men and women differ in their willingness to use automated cars? the influence of emotions across different age groups," Transportation Research Part A, vol. 94, pp. 374-385, 2016.

[30] L. A. Migliore, "Relation between big five personality traits and hofstede's cultural dimensions," Cross Cultural Management An International Journal, vol. 18, no. 1, pp. 38-54, 2013.

[31] R. W. Brislin, "Translation and content analysis of oral and written material," in Handbook of Cross-Cultural Psychology: Methodology, H. C. Triandis and J. W. Berry, Eds., pp. 389444, Allyn \& Bacon, Boston, MA, USA, 1980.

[32] K. Regmi, "Understanding the processes of translation and transliteration in qualitative research," International Journal of Qualitative Methods, vol. 9, no. 1, pp. 16-26, 2010.

[33] C. Richard, J. Yang, N. Song, F. Du, and K. Zhang, "Psychometric evaluation of Chinese-language 44-item and 10item big five personality inventories, including correlations with chronotype, mindfulness and mind wandering," PLoS One, vol. 11, no. 2, pp. 1-26, 2016.

[34] R. R. Mccrae and P. T. Costa, "Personality trait structure as a human universal," American Psychologist Journal, vol. 52, no. 5, pp. 509-516, 1997.
[35] M. W. Browne and R. Cudeck, "Alternative ways of assessing model fit," Sociological Methods \& Research, vol. 21, no. 2, pp. 230-258, 1992.

[36] L. t. Hu and P. M. Bentler, "Cutoff criteria for fit indexes in covariance structure analysis: conventional criteria versus new alternatives," Structural Equation Modeling: A Multidisciplinary Journal, vol. 6, no. 1, pp. 1-55, 1999.

[37] P. M. Bentler and D. G. Bonett, "Significance tests and goodness of fit in the analysis of covariance structures," Psychological Bulletin, vol. 88, no. 3, pp. 588-606, 1980.

[38] S. C. A. Domingues, J. B. Mendonça, R. Laranjeira, and E. M. Nakamura-Palacios, "Drinking and driving: a decrease in executive frontal functions in young drivers with high blood alcohol concentration," Alcohol, vol. 43, no. 8, pp. 657-664, 2009.

[39] T. W. Friedman, S. R. Robinson, and G. W. Yelland, "Impaired perceptual judgment at low blood alcohol concentrations," Alcohol, vol. 45, no. 7, pp. 711-718, 2011.

[40] M. Møller, S. Haustein, and C. G. Prato, "Profiling drunk driving recidivists in Denmark," Accident Analysis \& Prevention, vol. 83, pp. 125-131, 2015.

[41] E. J. Ogden and H. Moskowitz, "Effects of alcohol and other drugs on driver performance," Traffic Injury Prevention, vol. 5, no. 3, pp. 185-198, 2004.

[42] J. J. Fleiter and B. Watson, "Addressing the road trauma burden in China: exploring attitudes, behaviours, risk perceptions and cultural uniqueness," Accident Analysis \& Prevention, vol. 95, no. Pt B, pp. 326-333, 2016.

[43] R. M. Pendyala and R. Kitamura, "The rapid motorization of asia: implications for the future," Transportation, vol. 34, no. 3, pp. 275-279, 2007.

[44] B. Rammstedt and O. P. John, "Measuring personality in one minute or less: a 10-item short version of the big five inventory in English and German is," Journal of Research in Personality, vol. 41, no. 1, pp. 203-212, 2007.

[45] J. Luo and X. Y. Dai, "Development of the Chinese adjectives scale of big-five factor personality: theoretical framework and assessment reliability," Chinese Journal of Clinical Psychology, vol. 84, 2015. 